\title{
RHCG and TCAF1 promoter hypermethylation predicts biochemical recurrence in prostate cancer patients treated by radical prostatectomy
}

\author{
Siri H. Strand ${ }^{1}$, Michal Switnicki ${ }^{1}$, Mia Moller ${ }^{1}$, Christa Haldrup ${ }^{1}$, Tine M. \\ Storebjerg', ${ }^{1,3}$, Jakob Hedegaard ${ }^{1}$, Iver Nordentoft ${ }^{1}$, Soren Hoyer ${ }^{2}$, Michael \\ Borre $^{3}$, Jakob S. Pedersen ${ }^{1}$, Peter J. Wild ${ }^{4}$, Jong Y. Park ${ }^{5}$, Torben F. Orntoft ${ }^{1}$ and \\ Karina D. Sorensen ${ }^{1}$ \\ ${ }^{1}$ Department of Molecular Medicine, Aarhus University Hospital, Aarhus, Denmark \\ 2 Institute of Pathology, Aarhus University Hospital, Aarhus, Denmark \\ ${ }^{3}$ Department of Urology, Aarhus University Hospital, Aarhus, Denmark \\ ${ }^{4}$ Institute of Surgical Pathology, University Hospital Zurich, Zurich, Switzerland \\ ${ }^{5}$ Department of Cancer Epidemiology, Moffitt Cancer Center, Tampa, Florida, USA \\ Correspondence to: Karina D. Sorensen, email: kdso@clin.au.dk \\ Keywords: prostate cancer, DNA methylation, biomarker, diagnosis, prognosis \\ Received: December 14, 2016 Accepted: December 18, $2016 \quad$ Published: December 30, 2016
}

\section{ABSTRACT}

Purpose: The lack of biomarkers that can distinguish aggressive from indolent prostate cancer has caused substantial overtreatment of clinically insignificant disease. Here, by genome-wide DNA methylome profiling, we sought to identify new biomarkers to improve the accuracy of prostate cancer diagnosis and prognosis.

Experimental design: Eight novel candidate markers, COL4A6, CYBA, TCAF1 (FAM115A), HLF, LINC01341 (LOC149134), LRRC4, PROM1, and RHCG, were selected from Illumina Infinium HumanMethylation450 BeadChip analysis of 21 tumor ( $T$ ) and 21 non-malignant (NM) prostate specimens. Diagnostic potential was further investigated by methylation-specific qPCR analysis of 80 NM vs. 228 T tissue samples. Prognostic potential was assessed by Kaplan-Meier, uni- and multivariate Cox regression analysis in 203 Danish radical prostatectomy (RP) patients (cohort 1), and validated in an independent cohort of 286 RP patients from Switzerland and the U.S. (cohort 2).

Results: Hypermethylation of the 8 candidates was highly cancer-specific (area under the curves: 0.79-1.00). Furthermore, high methylation of the 2-gene panel RHCG-TCAF1 was predictive of biochemical recurrence (BCR) in cohort 1 , independent of the established clinicopathological parameters Gleason score, pathological tumor stage, and pre-operative PSA (HR (95\% confidence interval (CI)): 2.09 (1.26 - 3.46); $P=0.004)$, and this was successfully validated in cohort 2 (HR ( $95 \%$ CI): 1.81 (1.05 - 3.12); $P=0.032$ ).

Conclusion: Methylation of the RHCG-TCAF1 panel adds significant independent prognostic value to established prognostic parameters for prostate cancer and thus may help to guide treatment decisions in the future. Further investigation in large independent cohorts is necessary before translation into clinical utility.

\section{INTRODUCTION}

Prostate cancer is the most commonly diagnosed non-cutaneous malignancy in men in the Western world [1]. The diagnosis is based on elevated serum PSA (prostate-specific antigen), suspicious digital rectal examination and histopathologic evaluation of the sampled biopsies. Unfortunately, the currently available routine prognostic tools (mainly Gleason score (GS), serum PSA, and tumor stage) are unable to clearly distinguish aggressive from indolent disease at the time of diagnosis. Moreover, the lack of biomarkers for aggressive disease, 
combined with opportunistic PSA screening, has led to large scale overdiagnosis and overtreatment of clinically insignificant prostate cancers, thus new biomarkers are urgently needed.

Methylation of $\mathrm{CpG}$ dinucleotides in promoter regions is an essential mechanism of long-term gene silencing. In human malignancies, aberrant hypermethylation of promoter-associated $\mathrm{CpG}$ islands (CGIs) is a well-established mechanism for tumor suppressor gene (TSG) silencing [2]. While recurrent somatic mutations are rare in prostate cancer, aberrant promoter hypermethylation occurs early and more consistently in tumor development and progression, and thus constitutes a promising source for discovery of novel biomarkers. Indeed, DNA methylation alterations have shown significant potential as diagnostic as well as prognostic biomarkers for prostate cancer [3].

Here, we performed genome-wide DNA methylation profiling of 21 tumor (T) and 21 non-malignant (NM) prostate tissue specimens using the Illumina Infinium HumanMethylation450 BeadChip (450K array), and selected 8 novel methylation marker candidates associated to the promoter regions of 8 genes for further investigation: COL4A6 (collagen, type IV, alpha 6), CYBA (cytochrome b-245, alpha polypeptide), TCAF1 (TRPM8 channelassociated factor 1 (previously FAM115A)), HLF (hepatic leukemia factor), LINC01341 (long intergenic non-protein coding RNA 1341 (previously LOC149134)), LRRC4 (leucine rich repeat containing 4), PROM1 (prominin 1), and $R H C G$ (Rh family, C glycoprotein). Hypermethylation of all candidates was highly cancer-specific in surgical specimens as well as in diagnostic needle biopsy samples (DNBs, area under the curves (AUCs): 0.79-1.00). Finally, a 2-gene panel comprising RHCG and TCAF1 methylation was developed in the training cohort (203 Danish prostate cancer patients) and successfully validated in the validation cohort (286 Swiss/American prostate cancer patients) where it added significant independent prognostic value to routine clinicopathological parameters. This is the first study to show a prognostic biomarker potential for RHCG and TCAF1 methylation in prostate cancer.

\section{RESULTS}

\section{Identification and validation of candidate methylation markers}

To identify novel candidate markers for prostate cancer, global DNA methylation analysis was performed in $21 \mathrm{~T}, 12$ adjacent normal (AN), and 9 true normal (N) prostate tissue samples (Table $\mathrm{S} 1$ ), in addition to 3 malignant (PC3, LNCaP, 22rv1) and 2 NM (PrEC, $\mathrm{BPH} 1)$ prostate cell lines using the Illumina $450 \mathrm{~K}$ array. By multi-dimensional scaling analysis, tumor samples showed highly distinct and heterogenous methylation patterns compared to AN and $\mathrm{N}$ samples, which clustered tightly together (Figure 1A). No significant differential methylation was observed between $\mathrm{AN}$ and $\mathrm{N}$ samples
A

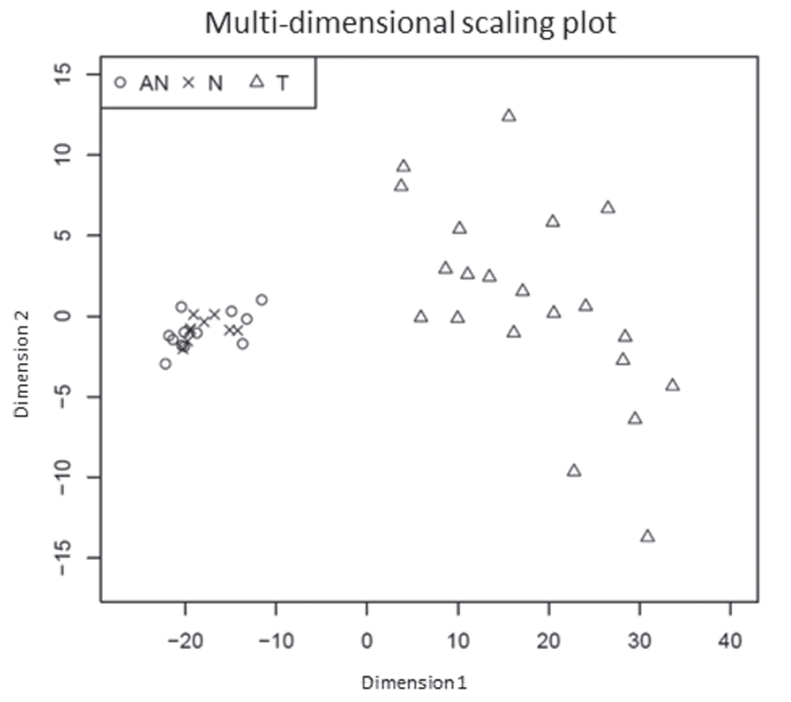

B

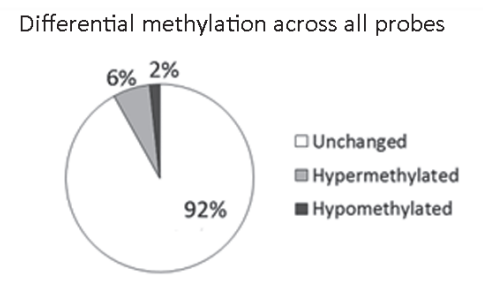

D

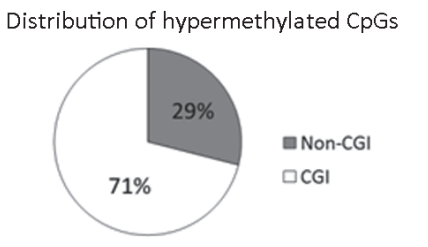

Figure 1: Differential methylation (T vs. NM) according to Illumina 450K array. A. Multi-dimensional scaling plot of samples included in $450 \mathrm{~K}$ analysis, based on the 10,000 most variable $\mathrm{CpG}$ sites across all samples. T samples $(N=21)$ : Triangles; AN samples $(N=12)$ : Circles; $N$ samples $(N=9)$ : Crosses. B.-E. Distribution of differential methylation (T vs. NM) according to the $450 \mathrm{~K}$ array. Differentially methylated $\mathrm{CpG}$ sites (DMCs) were defined as $\mathrm{CpG}$ sites with $\Delta \beta \geq|0.2|$ and adj. $P<0.05$. B. Differential methylation across all probes. C. Distribution of DMCs. D. Distribution of hypermethylated DMCs. E. Distribution of hypomethylated DMCs. 
(LIMMA differential methylation analysis [4], data not shown), which were thus pooled into one NM sample group. By comparing methylation in T vs. NM samples, we identified 37,763 differentially methylated $\mathrm{CpG}$ sites (DMCs: mean $\Delta \beta \geq|0.2|$, adj. $P<0.05$ ), the majority of which were hypermethylated $(N=29,748$, Figure 1B) and CGI-associated (Figure 1C, 1D). Conversely, most of the 8,015 DMCs displaying significant hypomethylation in tumors were located outside CGIs (Figure 1E). Thus, we observed cancer-specific hypermethylation of CGIs, whereas CpG sites outside CGIs were largely hypomethylated, consistent with previous reports of methylation patterns in cancer, including prostate cancer $[2]$.

To identify candidate biomarkers exhibiting highly cancer-specific differential methylation, we applied a strict threshold (mean $\Delta \beta \geq|0.55|$ ), generating a shortlist of the most differentially methylated CpGs $(N=324)$. Next, filtering for gene association (according to Illumina annotations) generated a final list of 259 top candidate DMCs (adj. $P<0.05$, mean $\Delta \beta>|0.55|$ ), associated to 163 genes (Figure 2A, Table S2). In addition to many novel candidates, this list contained several genes known to be frequently hypermethylated in prostate cancer (e.g. GSTP1, RARB [5]), supporting the validity of our results. From this list, 8 novel top candidate genes were selected based on their display of highly cancer-specific hypermethylation over multiple adjacent promoterassociated DMCs: COL4A6, CYBA, HLF, LINC0134 (LOC149234), LRRC4, PROM1, RHCG, and TCAF1 (FAM115A) (Figure 2B, S1 and S2, Table S3). None of these candidates have been previously investigated as potential prostate cancer methylation markers.

For technical validation of the $450 \mathrm{~K}$ array data, methylation levels of the 8 candidates were investigated by bisulfite sequencing (BS) in the 5 prostate cell lines. BS showed low methylation levels in NM cell lines (PrEC, BPH1) and high levels in malignant cell lines (PC3, LNCaP, 22rv1) for all 8 candidates, and fully corroborated the $450 \mathrm{~K}$ results for these cell lines (Figure S3). Notably, by both $450 \mathrm{~K}$ and BS analysis, we observed the same methylation patterns for NM vs. malignant
A

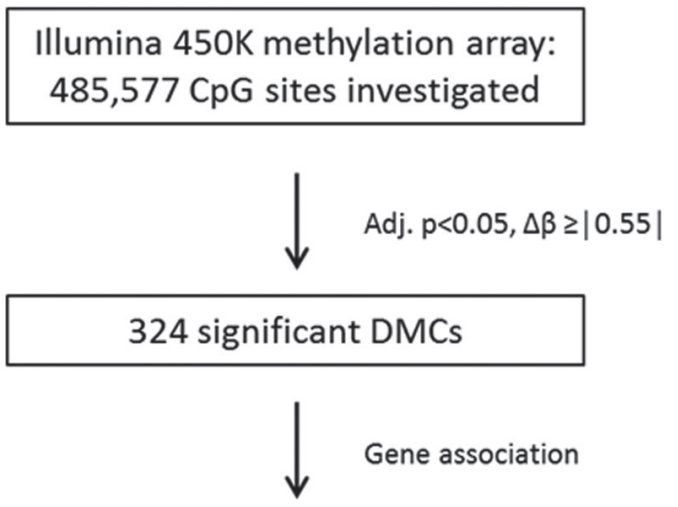

259 significant DMCs (163 genes)

Candidate gene selection
B

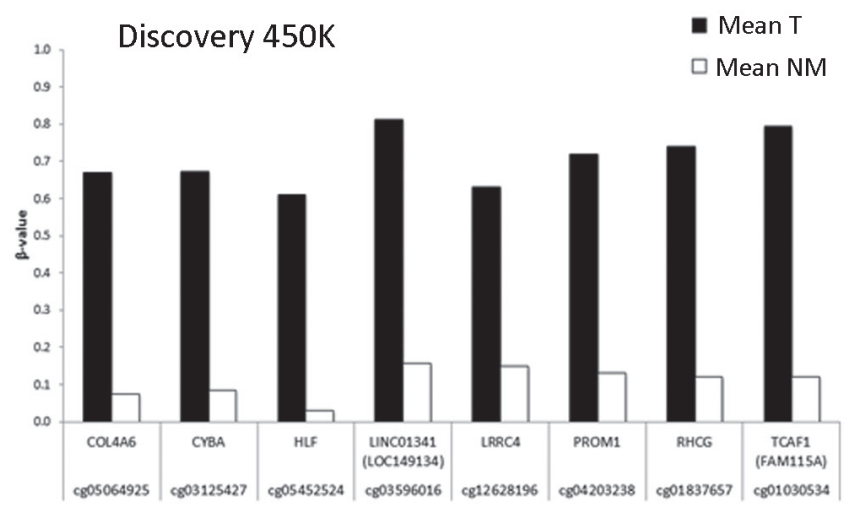

C

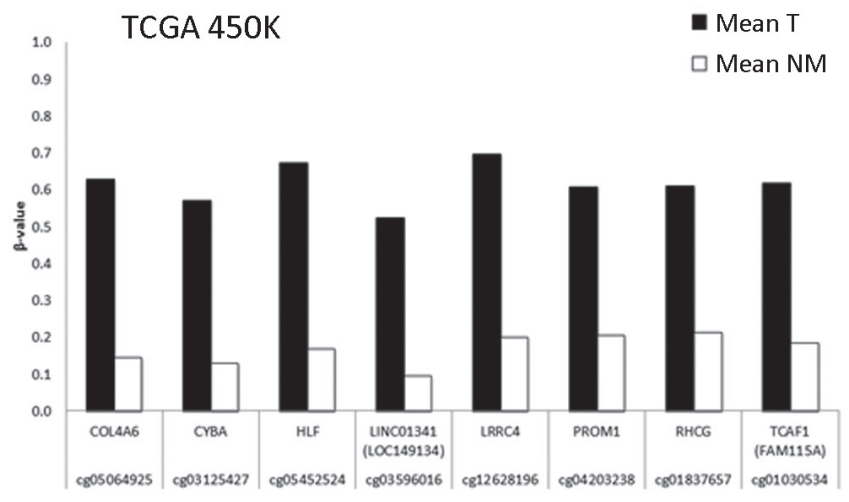

Figure 2: Selection of biomarker candidates. A: Candidate selection process. Of the 324 top candidate significant DMCs, 259 were associated to a total of 163 different genes. Eight genes were selected for further validation. B: Mean methylation of representative DMCs for each selected candidate in $\mathrm{T}$ and $\mathrm{NM}$ samples according to the discovery $450 \mathrm{~K}$ dataset ( $\mathrm{T}, N=21 ; \mathrm{NM}, N=21)$. C: Mean methylation of the same DMCs as in (B) for each candidate in T and NM samples according to the TCGA 450K dataset for prostate cancer (T, $N=297$; $\mathrm{NM}, N=34)$." . 
Table 1: Clinicopathological characteristics of patients in RP cohorts 1 and 2

\begin{tabular}{|c|c|c|}
\hline & $\begin{array}{c}\text { Cohort } 1 \\
T\end{array}$ & $\begin{array}{c}\text { Cohort } 2 \\
T\end{array}$ \\
\hline$N$ & 203 & 286 \\
\hline Age at RP, median (range) & $63(47-77)$ & $61(41-76)$ \\
\hline Unknown & $0(0.0 \%)$ & $5(1.7 \%)$ \\
\hline \multicolumn{3}{|l|}{ Pathological Gleason score } \\
\hline$<7, N(\%)$ & $96(47.3 \%)$ & $125(43.7 \%)$ \\
\hline$=7, N(\%)$ & $85(41.9 \%)$ & $135(47.2 \%)$ \\
\hline$>7, N(\%)$ & $22(10.8 \%)$ & $26(9.1 \%)$ \\
\hline \multicolumn{3}{|l|}{ Pathological T-stage (n) } \\
\hline$\leq \mathrm{pT} 2 \mathrm{c}, N(\%)$ & $131(64.5 \%)$ & $216(75.5 \%)$ \\
\hline$\geq \mathrm{pT} 3 \mathrm{a}, N(\%)$ & $72(35.5 \%)$ & $70(24.5 \%)$ \\
\hline \multicolumn{3}{|l|}{ Pre-operative PSA } \\
\hline PSA ng/ml, median (range) & $12.1(2.0-61.0)$ & $6.4(0.6-62.1)$ \\
\hline \multicolumn{3}{|l|}{ Surgical margin status } \\
\hline Negative, $N(\%)$ & $140(69.0 \%)$ & $72(25.2 \%)$ \\
\hline Positive, $N(\%)$ & $63(31.0 \%)$ & $34(11.9 \%)$ \\
\hline Unknown, $N(\%)$ & $0(0.0 \%)$ & $181(63.3 \%)$ \\
\hline \multicolumn{3}{|l|}{ Lymph node status } \\
\hline Positive, $N(\%)$ & $0(0.0 \%)$ & $0(0.0 \%)$ \\
\hline Negative, $N(\%)$ & $24(11.8 \%)$ & $23(8.0 \%)$ \\
\hline Unknown, $N(\%)$ & $179(88.2 \%)$ & $263(92.0 \%)$ \\
\hline $\begin{array}{l}\text { Median follow-up, months } \\
\text { (range) }\end{array}$ & $70(11-184)$ & $73(3-290)$ \\
\hline PSA recurrence, $\boldsymbol{N}(\%)$ & $85(41.9 \%)$ & $92(32.2 \%)$ \\
\hline
\end{tabular}

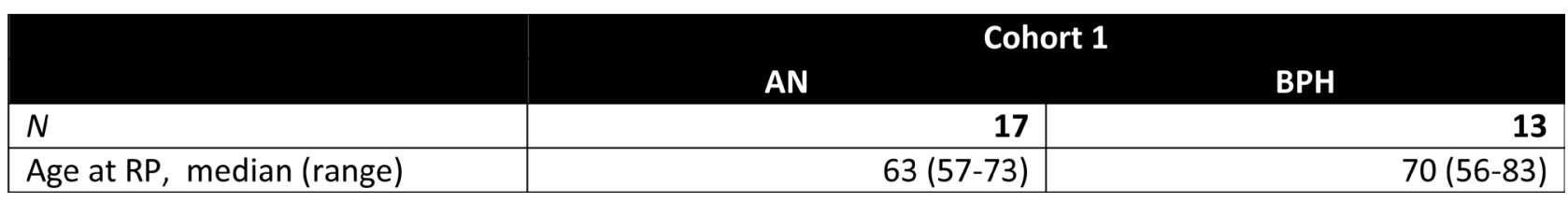

cell lines, as was observed for NM $v s$. malignant tissue specimens in the $450 \mathrm{~K}$ analysis. Moreover, we performed in-silico validation using $450 \mathrm{~K}$ data from The Cancer Genome Atlas (TCGA) [6, 7] for $297 \mathrm{~T}$ and $34 \mathrm{AN}$ prostate specimens, which independently confirmed prostate cancer-specific hypermethylation of all 8 genes (Figure 2C, Table S3). Finally, in order to investigate tissue specificity of our novel candidate markers, we used publicly available $450 \mathrm{~K}$ methylation array data from the Marmal-aid database [8], focusing on urological cancers and corresponding NM tissue (bladder cancer, $N=85$; NM bladder, $N=10$; kidney cancer, $N=244$; NM kidney, $N=$ 136). We found that hypermethylation of COL4A6, CYBA, LINC01341, and RHCG was highly prostate cancerspecific (Figure S4), suggesting particularly promising diagnostic potential for these genes.

Next, we investigated whether hypermethylation of our candidate biomarkers was associated with altered gene expression, using a small in-house (14 T, $12 \mathrm{NM})$ and the large TCGA (297 T, 34 NM) RNA-seq datasets. While the non-coding LINC01341 lacked annotation in both datasets, 6 of the remaining 7 candidate genes were downregulated in T vs. NM samples, consistent with epigenetic silencing through aberrant promoter hypermethylation (Figure S5, Table S3). TCAF1 was not significantly deregulated in the large TCGA dataset, yet we observed a modest but significant upregulation of this transcript in prostate cancer in the small dataset. Notably, aberrant hypermethylation of TCAF1 was specific to an intragenic CGI/shore region overlapping a putative alternative transcription start site (TSS), suggesting that hypermethylation of this region may stimulate transcription from the upstream TSS (TSS1, Figure $\mathrm{S} 1 \mathrm{H}$ ) in at least some prostate cancers.

Together, these data indicate that COL4A6, CYBA, HLF, LINC01341, LRRC4, PROM1, RHCG, and TCAF1 are frequent targets of hypermethylation in prostate cancer, and that aberrant promoter hypermethylation contributes to downregulation of 6 of these genes in this malignancy. 
A

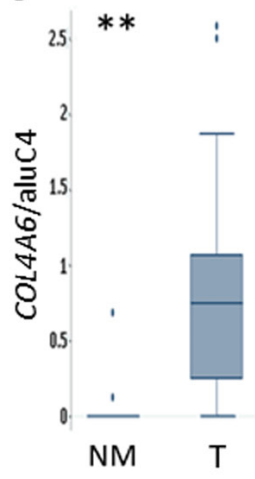

B
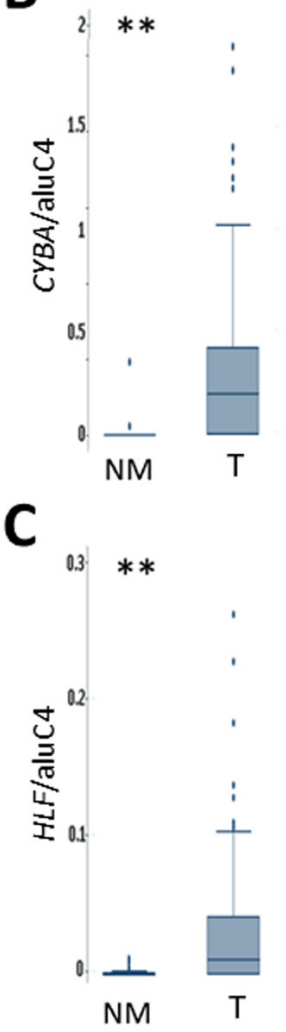

D

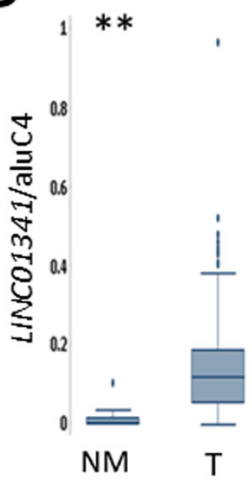

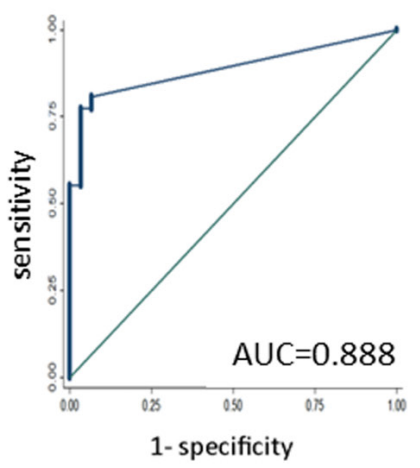
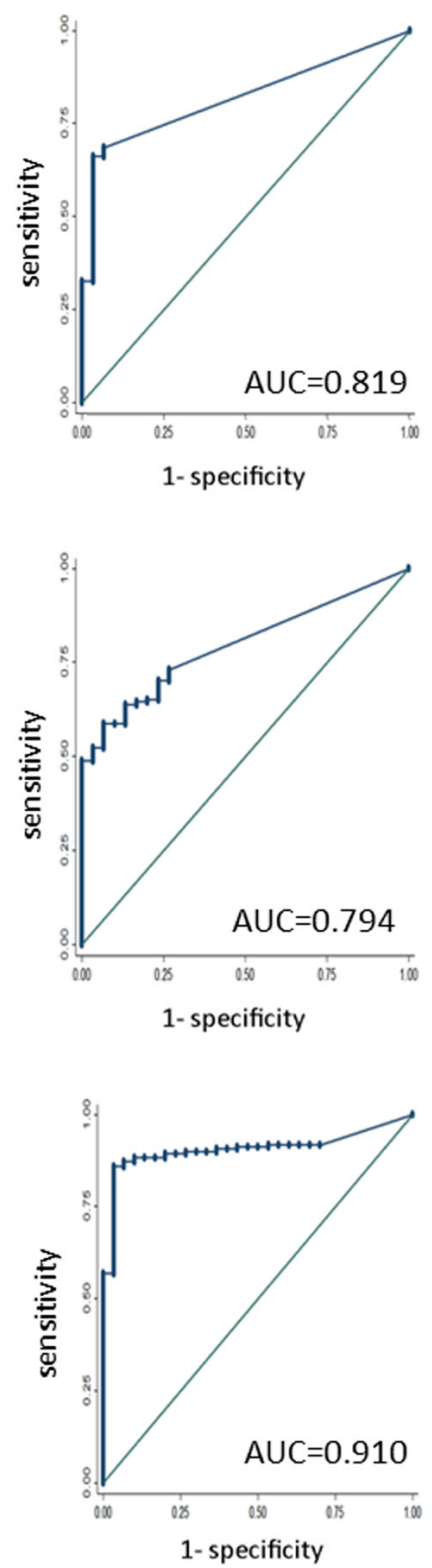

$\mathbf{E}$

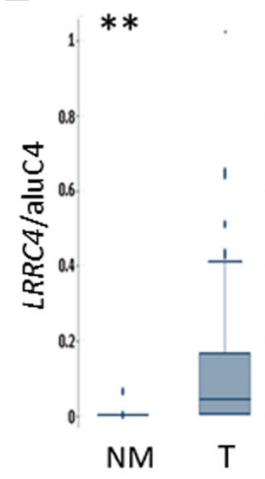

$\mathbf{F}$

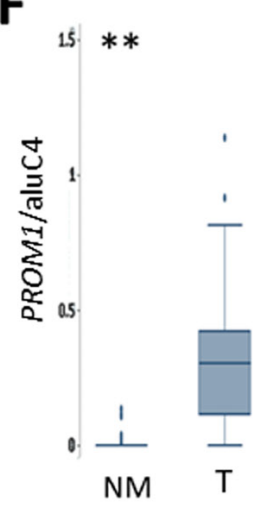

$\mathbf{G}$

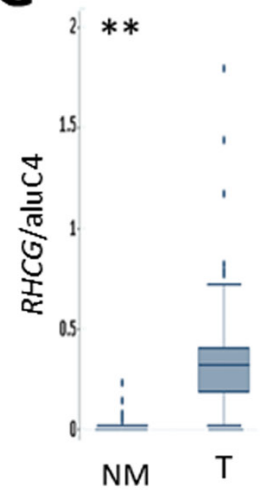

H

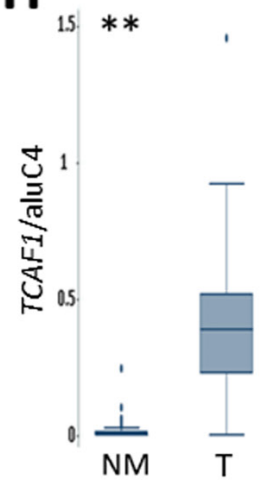

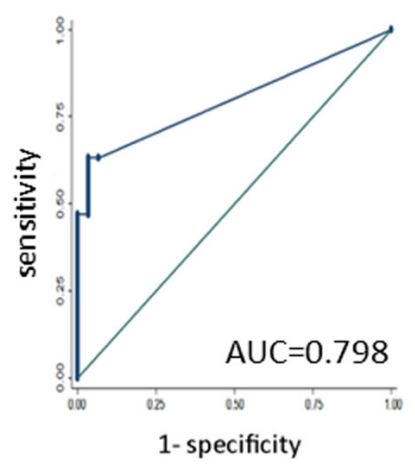
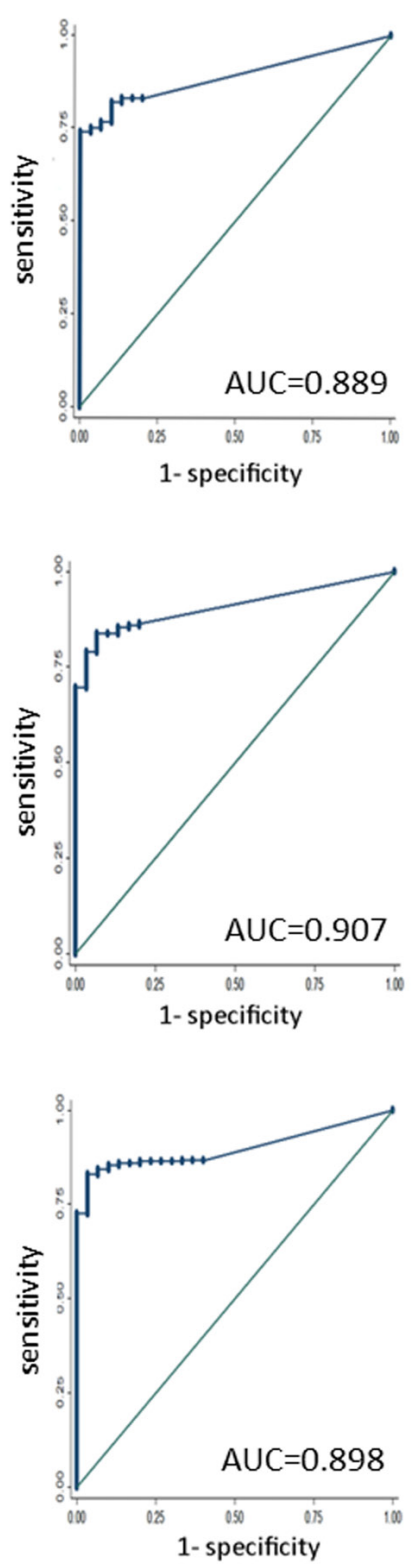

Figure 3: Diagnostic potential of candidate methylation markers in T, AN and BPH samples (cohort 1). ROC analysis of NM samples (AN and BPH, $N=30)$ vs. T samples $(N=203$ ). Left: Box plots of methylation levels in NM and T samples. $(* *) P<0.001$, rank-sum test. Right: ROC curves of data displayed in box plots. A. COL4A6. B. CYBA. C. HLF. D. LINC01341. E. LRRC4. F. PROM1. G. $R H C G$. H. TCAF1. 


\section{Diagnostic potential}

To investigate the diagnostic potential of our 8 candidates, methylation levels were examined in $203 \mathrm{~T}$ and $30 \mathrm{NM}$ prostate tissue samples (cohort 1, Table 1) using methylation-specific qPCR (qMSP). For all 8 loci, $\mathrm{T}$ samples displayed highly significant hypermethylation compared to NM samples (Figure 3) with AUCs ranging from $0.79(H L F)$ to 0.91 (LINC01341). At 96.7\% fixed specificity (Table S4), sensitivities ranged from $52.2 \%$ $(H L F)$ to $82.8 \%$ (TCAF1). To further examine the diagnostic potential, we performed qMSP analysis on 25 malignant and $50 \mathrm{NM}(25 \mathrm{AN}+25 \mathrm{~N})$ diagnostic needle biopsy (DNB) specimens. Again, highly significant cancer-specific hypermethylation was observed for all 8 candidates, with AUCs ranging from 0.97 (HLF) to 1.00 (PROM1, TCAF1) (Figure S6). At 96\% fixed specificity (Table S4), sensitivities ranged from $88 \%$ ( $H L F$, LINC01341) to $96 \%$ (COL4A6, TCAF1, LRRC4).

These results from qMSP analysis of both surgical and DNB specimens confirmed that all 8 genes are highly frequent targets of prostate cancer-specific aberrant hypermethylation, and that our qMSP assays can be used on DNBs.

\section{Prognostic potential of single candidates}

To investigate their possible prognostic potential in prostate cancer, methylation levels of each candidate was initially compared to routine clinicopathological factors in radical prostatectomy (RP) cohort 1 , as well as in an independent validation cohort including $286 \mathrm{RP}$ patients (cohort 2, Table 1). For all 8 candidates, a significant correlation between high methylation and at least one established adverse prognostic factor (high GS, advanced pathological tumor stage (pT), high pre-operative PSA) was observed in at least one cohort (Figure S7, Table S5). There were no significant correlations between methylation levels and age (Table S5).

To further assess the prognostic value of the 8 candidates, we investigated whether methylation levels were associated with time to biochemical recurrence (BCR) after RP. Initially, candidate gene methylation (as a continuous variable) was investigated in univariate Cox regression analysis in RP cohort 1. Here, COL4A6, PROM1, RHCG, and TCAF1 were significant predictors of BCR (adj. $P \leq 0.006$, Table S6). These results were subsequently tested and successfully validated by univariate Cox regression analysis in RP cohort 2 (adj. $P \leq$ 0.008 , Table $\mathrm{S} 6$ ). All routine clinicopathological variables were also significant predictors of BCR in univariate Cox regression analyses in both cohorts (Table S6), indicating that these are representative RP cohorts.

Next, in cohort 1, we performed multivariate Cox regression analysis including the 4 candidates significant in univariate analysis, in addition to GS, pT stage, surgical margin (SM) status, and pre-operative PSA. Here, both $R H C G$ and TCAF1 were significant predictors of BCR, independent of routine clinicopathological parameters (RHCG hazard ratio (HR) $(95 \%$ confidence interval (CI)): $1.60(1.07-2.38), P=0.021 ;$ TCAF1 HR $(95 \% \mathrm{CI})$ : 2.82 (1.54-5.17), $P=0.001$, Table 2). The independent prognostic potentials of $R H C G$ and TCAFl were subsequently tested in the validation cohort, where both candidates were significant adverse predictors of BCR, independent of clinicopathological variables ( $R H C G \mathrm{HR}$ (95\% CI): 1.55 (1.23 - 1.94), adj. $P=0.001$; TCAF1 HR (95\% CI): 1.48 (1.18 - 1.85), adj. $P=0.001$, Table 2). Thus, RHCG and TCAF1 hypermethylation, in addition to routine clinicopathological parameters, were independent predictors of $\mathrm{BCR}$ in multivariate analysis in both RP cohorts.

We applied Harrell's C-index to estimate the predictive accuracy of the multivariate model. In cohort 1, adding RHCG and TCAF1 methylation to a model of clinicopathological factors (GS, pT, SM, pre-op. PSA) improved the $\mathrm{C}$-index from 0.769 to 0.782 , whereas in cohort 2, adding RHCG and TCAF1 methylation to the clinicopathological model (GS, pT, pre-op. PSA) increased the C-index from 0.703 to 0.718 , indicating that $R H C G$ and $T C A F 1$ methylation improved the predictive accuracy in both cohorts and thus carries prognostic potential beyond that of routinely used clinicopathological factors.

In order to simplify test interpretation in the clinic, biomarkers are often analyzed as dichotomized variables. Thus, using cohort 1 for training, RHCG and TCAF1, respectively, were divided into high- or low- methylation by receiver operating characteristics (ROC) analysis (BCR status at 36 months follow-up) and analyzed for their ability to predict BCR. In cohort 1, high methylation of each candidate significantly predicted BCR in both Kaplan-Meier (adj. $P=0.0001$, Figure 4), uni- and multivariate Cox regression analyses (adj. $P<0.05$, Tables S7-S9).

Using the numerical cut-offs defined in cohort 1 , we subsequently tested and validated the prognostic potential of dichotomized $R H C G$ and TCAF1 in KaplanMeier (adj. $P<0.013$, Figure 4), uni- and multivariate Cox regression analyses (adj. $P \leq 0.04$, Tables $\mathrm{S} 7-\mathrm{S} 9$ ) in cohort 2. Thus, dichotomized RHCG and TCAF1 methylation, in addition to routine clinicopathological parameters, were independent predictors of BCR in both cohorts. Moreover, adding these methylation candidate markers to the clinicopathological models increased the $\mathrm{C}$-indices in both cohorts (Table S8 and S9). Together, these results indicate that dichotomized RHCG and TCAF1 improved the predictive accuracy beyond routine clinicopathological prognostic variables in both cohorts. 


\section{Prognostic methylation panel}

Prostate cancer is a highly heterogeneous disease at the molecular level, and multi-gene panels may improve the robustness of individual markers [9]. Thus, we generated a 2-gene panel consisting of dichotomized $R H C G$ and TCAFl (termed $\mathrm{d} R H C G-T C A F 1$ ), where patients with high methylation of both genes were classified into the high-methylation group, and the remaining patients into the low-methylation group. In cohort 1 , high methylation of $\mathrm{d} R H C G-T C A F 1$ significantly predicted $\mathrm{BCR}$ in univariate $(p<0.001$, Table 3$)$ and multivariate Cox regression analyses (HR (95\% CI): 2.09 (1.26-3.46), $P=0.004$, Table 3), as well as in KaplanMeier analysis $(p<0.0001$, Figure 5A). The prognostic potential of $\mathrm{d} R H C G-T C A F 1$ was subsequently validated in cohort 2, where it was a significant adverse predictor in both univariate $(P=0.004$, Table 3$)$ and multivariate (HR (95\% CI): 1.81 (1.05-3.12), $P=0.032$, Table 3) Cox regression analyses, as well as in Kaplan-Meier analysis $(P$
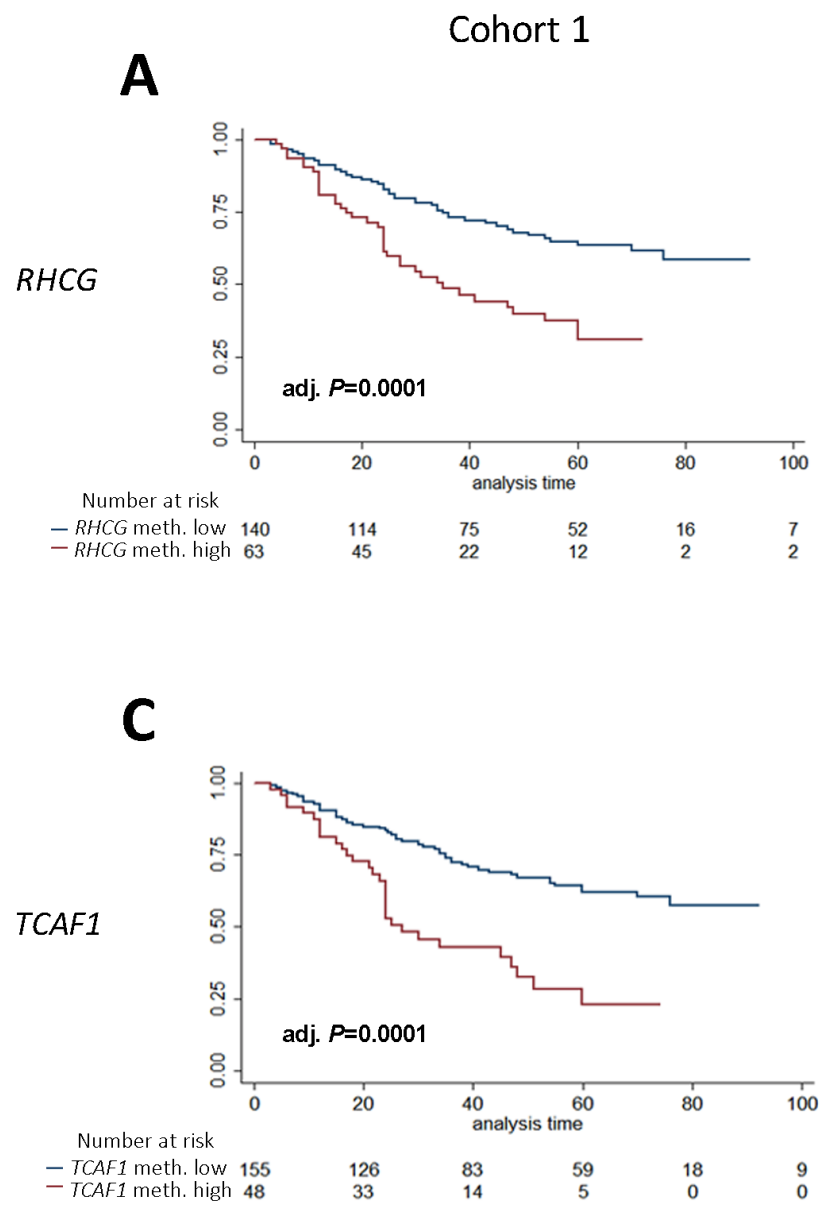

$=0.0008$, Figure 5B). In cohort 1 , the $\mathrm{C}$-index increased from 0.769 to 0.777 when $\mathrm{d} R H C G-T C A F 1$ methylation was added to the clinicopathological model, whereas in cohort 2, it increased from 0.703 to 0.717 by addition of $\mathrm{d} R H C G-T C A F 1$ methylation, indicating improved model performance by adding the 2-gene panel to the established prognostic parameters. Thus, $\mathrm{d} R H C G-T C A F 1$ methylation, together with all available clinicopathological parameters, independently predicted $\mathrm{BCR}$ in multivariate analysis in both RP cohorts.

Next, we investigated the prognostic potential of a trichotomized methylation model ( $\mathrm{t} R H C G-T C A F 1)$, where patients in cohort 1 were stratified into 3 groups based on whether they had high methylation of both candidates (high-methylation), one candidate (intermediatemethylation), or neither candidate (low-methylation). By Kaplan-Meier analysis, patients in the high-methylation group had significantly higher risk of BCR than patients in the 2 remaining groups, where patients with low methylation also had the lowest risk of BCR $(P=0.0001$, Figure 6A). This was successfully validated in cohort 2
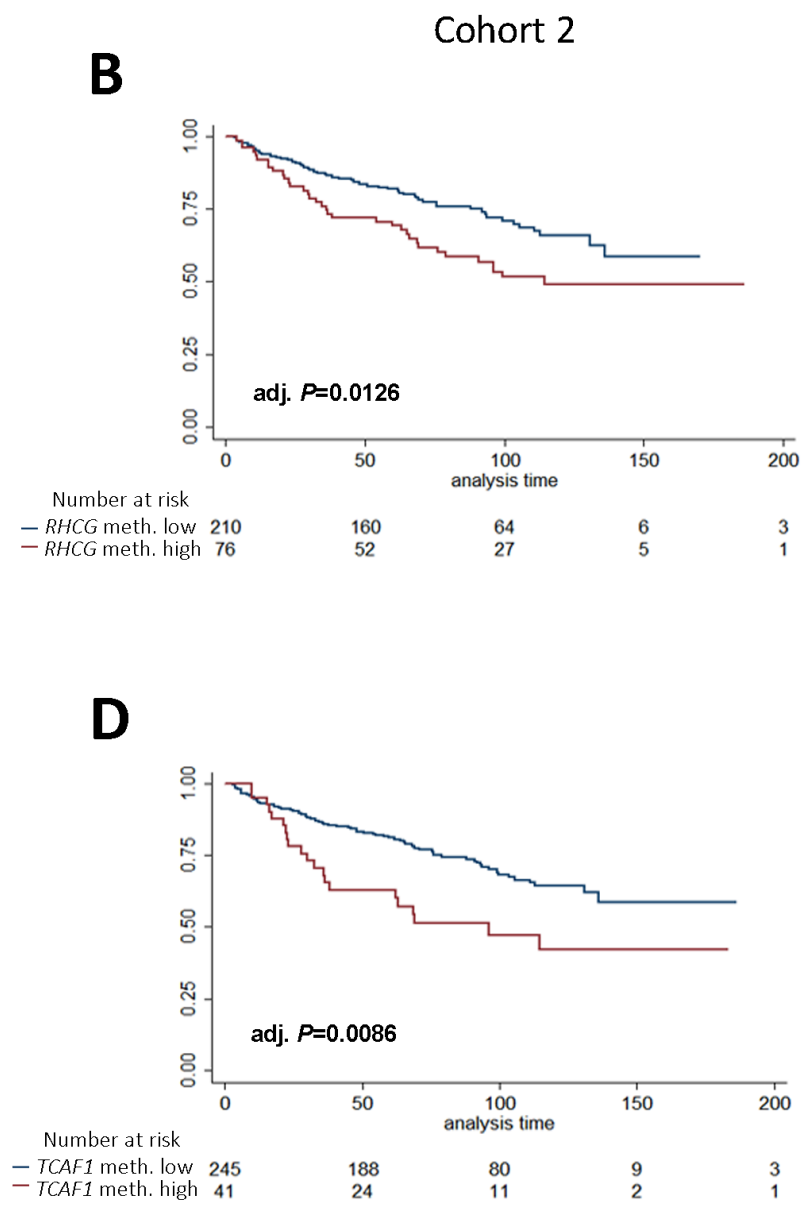

Figure 4: Kaplan-Meier analysis of association between methylation levels of candidate biomarkers and time to BCR after RP. Patients were divided into high- or low methylation groups based on ROC analysis of BCR status (36 months after RP, cohort 1). A, B: RHCG analyzed in cohort 1 (A) and cohort 2 (B). C, D: TCAF1 analyzed in cohort 1 (C) and cohort 2 (D). P-values from log-rank test, adjusted according to the Hochberg procedure. 
( $P=0.0050$, Figure 6B). Moreover, when comparing the high and low-methylation groups only, high methylation was a significant predictor of BCR in uni- and multivariate Cox regression analyses in cohort 1 (Table S10), and these results were successfully validated in cohort 2 (Table S10), suggesting that prostate cancer patients can be stratified into clinically relevant subgroups based on $R H C G$ and TCAF1 methylation. Notably, 3 years after surgery, $61 \%$ of patients with high methylation of the RHCG-TCAF1 panel had suffered BCR, compared to $23 \%$ of patients with low methylation in cohort 1 . Likewise, in cohort 2 , $35 \%$ of patients with high $R H C G-T C A F 1$ methylation had suffered BCR 3 years after surgery, compared to $23 \%$ of patients with low methylation. In summary, these results suggest that high methylation of the 2-gene panel $R H C G$ $T C A F 1$ is a significant adverse predictor of BCR after RP, independent of routine clinicopathological parameters.

A

Cohort 1

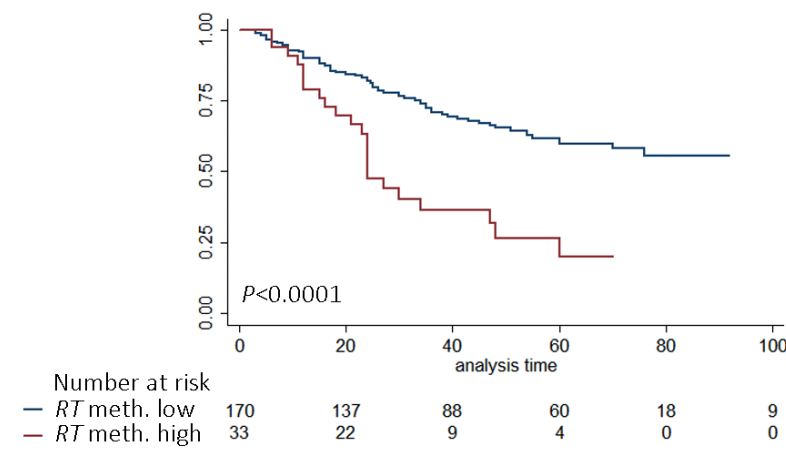

\section{DISCUSSION}

Here, we identified COL4A6, CYBA, HLF, LINC01341, LRRC4, PROM1, RHCG, and TCAF1 as novel targets of frequent hypermethylation in prostate cancer. Cancer-specific hypermethylation was observed for all candidates in both surgical and DNB specimens, suggesting promising diagnostic biomarker potential. Furthermore, we generated a new 2-gene panel comprising RHCG and TCAF1 methylation, which predicted BCR in two RP cohorts from Denmark, Switzerland, and the U.S. independently of routine clinicopathological parameters. This is the first study to demonstrate a significant prognostic value of RHCG and TCAF1 hypermethylation in prostate cancer.

In addition to technical validation by $\mathrm{BS}$, hypermethylation in prostate cancer of our eight candidates was confirmed in the large external TCGA dataset (450K),

B

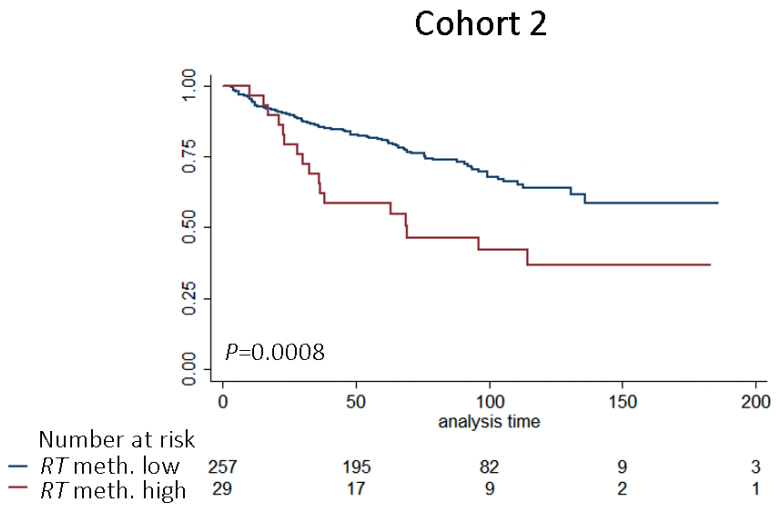

Figure 5: Kaplan-Meier analysis of the association between methylation levels of the marker panel $\mathrm{d} R H C G$-TCAF1 and time to BCR after RP. Patients in cohort 1 (A) and cohort 2 (B) divided into high-and low methylation groups: High: High methylation of both candidates. Low: High methylation of only one, or low methylation of both candidates. RT: RHCG-TCAF1 panel. P-values from log-rank test.

A

Cohort 1

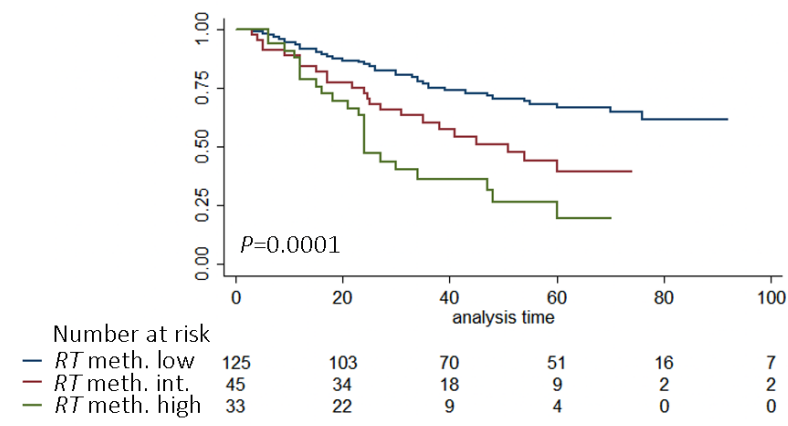

B

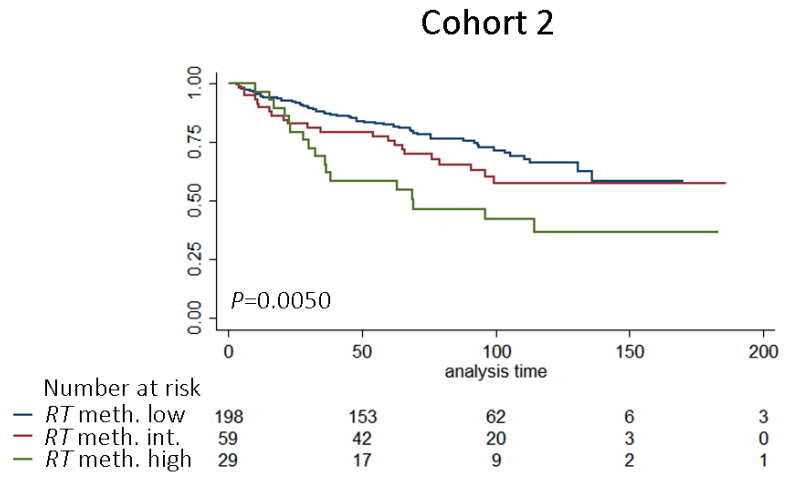

Figure 6: Kaplan-Meier analysis of the association between methylation levels of the marker panel tRHCG-TCAF1 and time to BCR after RP. Patients in cohort 1 (A) and cohort 2 (B) divided into high-, low- and intermediate methylation groups. High: High methylation of both candidates. Low: Low methylation of both candidates. Intermediate: High methylation of one candidate only. RT: RHCG-TCAF1 panel. $P$-values from log-rank test. 
Table 2: Multivariate analysis.

\begin{tabular}{|c|c|c|c|}
\hline \multicolumn{4}{|c|}{ Training cohort ( $N=203$ ) } \\
\hline \multicolumn{4}{|c|}{ Multivariate } \\
\hline Variable & $\mathrm{HR}(95 \% \mathrm{Cl})$ & $P$ & $\operatorname{adj} . P$ \\
\hline RHCG (cont.) & $1.60(1.07-2.38)$ & 0.021 & NA \\
\hline TCAF1 (cont.) & $2.82(1.54-5.17)$ & 0.001 & NA \\
\hline Tumor stage (pT2 vs. pT3-4) & $2.49(1.44-4.30)$ & 0.001 & NA \\
\hline Gleason score (<7 vs. $\geq 7$ ) & $1.86(1.16-2.96)$ & 0.009 & NA \\
\hline Pre-op. PSA (<10 vs. $\geq 10$ ) & $3.07(1.89-5.00)$ & $<0.001$ & NA \\
\hline Surgical margin (neg. vs. pos.) & $2.07(1.27-3.37)$ & 0.003 & NA \\
\hline
\end{tabular}

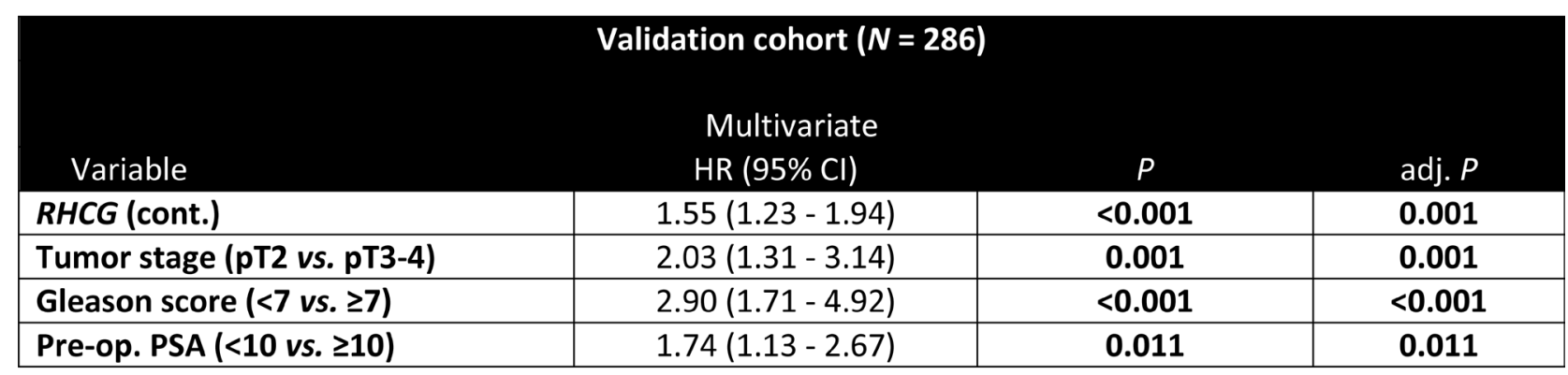

\begin{tabular}{|c|c|c|c|}
\hline \multicolumn{4}{|c|}{ Validation cohort $(N=286)$} \\
\hline \multicolumn{4}{|c|}{ Multivariate } \\
\hline Variable & $\mathrm{HR}(95 \% \mathrm{Cl})$ & $P$ & adj. $P$ \\
\hline TCAF1 (cont.) & $1.48(1.18-1.85)$ & 0.001 & 0.001 \\
\hline Tumor stage (pT2 vs. pT3-4) & $2.01(1.29-3.11)$ & 0.002 & 0.002 \\
\hline Gleason score (<7 vs. $\geq 7$ ) & $2.85(1.69-4.82)$ & $<0.001$ & $<0.001$ \\
\hline Pre-op. PSA (<10 vs. $\geq 10$ ) & $1.72(1.12-2.64)$ & 0.013 & 0.013 \\
\hline
\end{tabular}

Multivariate Cox regression analysis of methylation marker candidates and clinicopathological variables analyzed as continuous variables with stepwise backwards selection in cohort 1 (top) and of RHCG and TCAF1 in cohort 2 (middle and bottom). Only candidate genes significant in cohort 1 (training) were tested in cohort 2 (validation). Bold: $P<0.05$. NA, not applicable.

further supporting their diagnostic potential. Moreover, as proof of principle, we demonstrated that our qMSP assays can be used on DNBs despite scarce sample amounts. While a molecular diagnostic test that can detect histologically verified prostate cancer in DNBs might have limited clinical utility, detection of methylationbased cancer field effects in morphologically normal prostate biopsies may be used to guide the need for repeat biopsy in men with exclusively cancer-negative DNBs but persistently elevated PSA, which remains a major clinical challenge $[10,11]$. Although we did not detect significant differential methylation between $\mathrm{AN}$ and $\mathrm{N}$ samples in this study, our analysis could be limited by the relatively small sample size. Further studies are needed to investigate the possible existence of epigenetic cancer field effects for our candidate genes. Future studies should also investigate whether our novel candidate markers are detectable in blood or urine samples, e.g. as circulating cell-free tumor DNA methylation, which could facilitate development of minimally or non-invasive testing for prostate cancer.

Here, using two large prostate cancer patient cohorts, we identified and independently validated $R H C G, T C A F 1$, and the 2-gene panel RHCG-TCAF1 as novel independent adverse predictors of BCR after RP. Prior to this, only a few candidate methylation markers have demonstrated prognostic potential in more than one prostate cancer cohort by multivariate analysis adjusting for established clinicopathological parameters [3]. These are PITX2 [12, 13], GABRE miR-452 miR-224 [14], C1orf114 (CCDC181) [9], and the panels C1orf114/ HAPLN3 and AOX1/C1orf114/HAPLN3 [9]. Further studies, using large independent cohorts with long follow- 
Table 3: Uni- and multivariate Cox regression analysis of the dichotomized $R H C G-T C A F 1$ panel

\begin{tabular}{|c|c|c|c|c|c|c|c|}
\hline \multicolumn{8}{|c|}{ Training cohort ( $N=203$ ) } \\
\hline \multirow[b]{2}{*}{ Variable } & \multicolumn{3}{|c|}{ Univariate } & \multicolumn{4}{|c|}{ Multivariate } \\
\hline & $\mathrm{HR}(95 \% \mathrm{Cl})$ & $P$ & C-index & $\mathrm{HR}(95 \% \mathrm{Cl})$ & $P$ & C-index ${ }^{a}$ & C-index ${ }^{b}$ \\
\hline $\mathrm{dRHCG-TCAF1}$ & $2.82(1.74-4.59)$ & $<0.001$ & 0.581 & $2.09(1.26-3.46)$ & 0.004 & \multirow{5}{*}{0.777} & \\
\hline Tumor stage (pT2 vs.pT3-4) & $4.46(2.86-6.97)$ & $<0.001$ & 0.677 & $2.74(1.66-4.55)$ & $<0.001$ & & \multirow{4}{*}{0.769} \\
\hline Gleason score (<7 vs. $\geq 7$ ) & $2.09(1.32-3.30)$ & 0.002 & 0.579 & $1.87(1.18-2.97)$ & 0.007 & & \\
\hline Pre-op. PSA (< 10 vs. $\geq 10$ ) & $2.73(1.59-4.71)$ & $<0.001$ & 0.600 & $2.72(1.56-4.71)$ & $<0.001$ & & \\
\hline Surgical margin (neg. vs. pos.) & $3.47(2.25-5.36)$ & $<0.001$ & 0.663 & $2.49(1.54-4.05)$ & $<0.001$ & & \\
\hline
\end{tabular}

\begin{tabular}{|c|c|c|c|c|c|c|c|}
\hline \multicolumn{8}{|c|}{ Validation cohort $(N=286)$} \\
\hline & \multicolumn{3}{|c|}{ Univariate } & \multicolumn{4}{|c|}{ Multivariate } \\
\hline Variable & $\mathrm{HR}(95 \% \mathrm{Cl})$ & $P$ & C-index & $\mathrm{HR}(95 \% \mathrm{Cl})$ & $\rho$ & C-index ${ }^{a}$ & C-index ${ }^{b}$ \\
\hline dRHCG-TCAF1 & $2.19(1.29-3.72)$ & 0.004 & 0.550 & $1.81(1.05-3.12)$ & 0.032 & \multirow{4}{*}{0.717} & \\
\hline Tumor stage (pT2 vs. pT3-4) & $3.16(2.09-4.78)$ & $<0.001$ & 0.629 & $2.05(1.33-3.17)$ & 0.001 & & \multirow{3}{*}{0.703} \\
\hline Gleason score (<7 vs. $\geq 7$ ) & $3.53(2.15-5.81)$ & $<0.001$ & 0.638 & $2.81(1.68-4.69)$ & $<0.001$ & & \\
\hline Pre-op. PSA (< 10 vs. $\geq 10$ ) & $2.51(1.66-3.79)$ & $<0.001$ & 0.593 & $1.62(1.05-2.49)$ & 0.028 & & \\
\hline
\end{tabular}

Analyses performed in cohort 1 (top) and 2 (bottom). Bold: $P<0.05$. ${ }^{a}$ Model including all variables significant in multivariate analysis. ${ }^{b}$ Model including clinicopathological variables only.

up, are needed to assess the clinical utility of $R H C G$ and $T C A F 1$ as well as of the previously identified prognostic methylation marker candidates. These candidates should be investigated individually, as well as in combinations, to identify their true prognostic potential.

Current prognostic prostate cancer classification systems rely on histopathological criteria, which cannot accurately predict whether a tumor will progress to clinically relevant disease or remain indolent. Novel biomarkers that enable distinction between aggressive and indolent cancer at the time of diagnosis could improve patient management significantly, e.g. by allowing active surveillance of low-risk patients and immediate treatment of high-risk patients. While the incremental gain in prognostic information obtained by adding parameters to existing models is often modest [15], the increased C-indices presented here nevertheless represent meaningful improvements to the model comprising only clinicopathological parameters [16]. The full multivariate model including the dichotomized methylation marker panel $\mathrm{d} R H C G-T C A F 1$ provided a C-index of 0.777 in cohort 1 and 0.717 in cohort 2. C-index analysis in reduced models, i.e. leaving out one variable at a time, demonstrated modest $\mathrm{C}$-index contributions for any single variable (range 0.008-0.028 in cohort 1 and 0.011-0.032 in cohort 2), corresponding to a maximum contribution of $3.6 \%$ and $5.2 \%$ in cohort 1 and 2, respectively (data not shown). Moreover, the observed increments in C-index for our novel 2-gene methylation marker panel are comparable to those reported for other prognostic DNA methylation marker candidates $[9,13,14]$, as well as for prognostic gene expression signatures that have been developed into commercial tests for prostate cancer, i.e. Decipher [17], Oncotype [18] and Prolaris [19].

Furthermore, as cancer-specific hypermethylation was detectable in DNBs, our results suggest that RHCG$T C A F 1$ has the potential to improve the accuracy of prostate cancer prognosis at the time of diagnosis, where only pre-operative clinicopathological parameters are available (biopsy-based GS, clinical tumor stage, and pre-operative PSA). Notably, more than $50 \%$ of prostate tumors are upstaged and/or upgraded after RP [20], further stressing the need for improved prognostic markers at an early stage. Thus, it is likely that our methylation marker candidates would contribute relatively more independent prognostic information in biopsy specimens at the time of diagnosis, than shown here for RP samples, and thus potentially could be used to guide treatment decisions in the future. Moreover, our qMSP assays are simple and cost-effective and as such would be easy to incorporate into routine diagnostic practice.

The potential functional roles of our 8 candidate methylation markers in prostate physiology and malignancy are largely unknown. Here, we found that expression of 6 of these genes was significantly downregulated in prostate cancer, consistent with epigenetic silencing and suggesting a possible function in prostate carcinogenesis and/or tumor progression. More specifically, we observed promoter hypermethylation and downregulation of $R H C G$ in prostate tumors, which is consistent with previous reports of $R H C G$ downregulation in kidney and oesophagal carcinomas [21, 22]. RHCG encodes an epithelial ammonia transporter that is widely expressed in the kidney, liver and intestinal tract, as well as in male reproductive organs, where it contributes to multiple components of fertility [23]. The role of $R H C G$ in the normal and cancerous prostate, however, is unknown and further studies are warranted.

$T C A F 1$ was recently identified as a positive regulator of TRPM8, an ionotropic testosterone receptor highly expressed in various organs, including the prostate $[24,25]$, but its exact function in the prostate is unknown $[25,26]$. According to our analysis, TCAF1 expression was unaltered or modestly upregulated in localized prostate tumors compared to NM prostate tissue samples, consistent with a previous study [24]. Several studies have 
reported that intragenic DNA methylation, as we observed for $T C A F 1$ in prostate cancer, is involved in alternative TSS-regulation in normal and malignant tissue [27]. Moreover, gene body methylation is a feature of actively transcribed genes [28]. Future studies should investigate TCAF1 methylation and potential isoform-specific expression patterns in NM and malignant prostate cells to elucidate its possible role in prostate cancer tumorigenesis and progression.

We observed cancer-specific promoter hypermethylation and downregulation of PROMI (CD133) expression, which is consistent with reports of PROM1 downregulation in several malignancies, including prostate cancer [29, 30]. PROM1 encodes a transmembrane glycoprotein widely used as a stem cell marker, but its potential role in prostate cancer is unknown. We also observed cancer-specific promoter hypermethylation and downregulation of $C O L 4 A 6$, which encodes a subunit of the epithelial basement membrane protein collagen IV. Our observations are consistent with reports of $C O L 4 A 6$ downregulation in a range of cancers, including prostate cancer [31]. Cancer-specific hypermethylation and transcriptional downregulation was also observed for $C Y B A, H L F$, and $L R R C 4$. CYBA encodes a subunit of an NADPH oxidase, whereas $H L F$ encodes a transcriptional activator of the proline and acidic-rich (PAR) protein family. LRRC4 is a proposed TSG involved in nervous system development and differentiation [32], and was reported to be hypermethylated and downregulated in gliomas [33]. However, the roles of these genes in prostate physiology and malignancy are unknown. Finally, we observed cancer-specific promoter hypermethylation of the verified but uncharacterized long non-coding RNA LINC01341 [34]. Unfortunately, no expression data was available for LINC01341 in the investigated datasets. Thus, further studies are needed to elucidate the possible role of LINC01341 in normal and malignant prostate biology.

There are some limitations to the present study. The prognostic analyses for our candidates were based on RP specimens, and while it could be useful to identify highrisk patients in need of adjuvant therapy post-RP (e.g. radiation treatment or androgen deprivation), there are currently no established adjuvant lines of treatment for patients after RP. Thus, future studies should evaluate the prognostic potential of these novel candidate methylation markers in DNBs, and thus investigate whether they can predict prostate cancer aggressiveness at the time of diagnosis and thereby help guide treatment decisions.

Moreover, the RP cohorts used for analysis of prognostic biomarker potential were of moderately different compositions, with patients in cohort 1 suffering more BCRs and having tumors with higher $\mathrm{pT}$ stage and higher median pre-operative PSA compared to patients in cohort 2 (Table 1). Nevertheless, all routine clinicopathological variables (GS, pT, pre-op. PSA) were significant predictors of BCR in uni- and multivariate Cox regression analysis in both cohorts, strongly indicating that both constitute representative RP cohorts. Moreover, our RHCG-TCAF1 panel was significant in multivariate analysis in both cohorts, using the exact numerical dichotomization/trichotomization cut-points derived from cohort 1 (training cohort), thus indicating the robustness of our methylation marker candidates.

Another limitation to our study is the lack of SM status information for most patients in cohort 2, thus this parameter was excluded from multivariate analysis in this cohort. Nevertheless, SM status was a significant predictor of BCR in uni- and multivariate Cox regression analysis in cohort 1 , as were our methylation marker candidates, further supporting their strength as independent prognostic biomarkers for prostate cancer. Furthermore, the use of $\mathrm{BCR}$ as end-point for survival analysis constitutes another possible limitation. BCR may result from either nonradical surgery, or micro metastatic disease manifesting prior to surgery, and is only a surrogate marker for tumor aggressiveness. Thus, future studies should investigate our novel methylation marker candidates in relation to more clinically relevant endpoints, such as metastatic progression or prostate cancer-specific mortality. Due to the slow progression of prostate cancer, such studies would require large cohorts with $>15$ years of follow-up [35]. Finally, while the cohorts used in this study included patients of European descent, further studies are needed to investigate if our findings can be extended to other ethnic groups.

In conclusion, we identified and validated 8 novel methylation marker candidates for prostate cancer diagnosis. We also identified and validated the prognostic potential of a new 2-gene methylation marker panel TCAF1-RHCG, which predicted time to BCR independently of established clinicopathological parameters in 2 RP cohorts. The actual clinical utility of these novel candidate methylation markers for prostate cancer diagnosis and prognosis should be further investigated in large independent cohorts with long follow-up and clinically relevant end-points. Moreover, an important future task will be to investigate whether methylation of RHCG and TCAF1 can also predict prostate cancer aggressiveness at the time of diagnosis based on analysis of DNBs or even liquid biopsies, in order to guide treatment decisions.

\section{MATERIALS AND METHODS}

\section{Infinium HumanMethylation450 BeadChip}

A total of $21 \mathrm{~T}, 14 \mathrm{AN}, 9 \mathrm{~N}$ prostate tissue samples (Table S1), and 5 cell lines (BPH1, 22rv1, LNCaP, PC3, and PrEC; Table S11) were analyzed by the Infinium 
HumanMethylation450 BeadChip (Illumina, San Diego, CA). Bisulfite conversion and genome-wide methylation analysis was conducted as a commercial service by The Genome Centre, Barts and the London School of Medicine and Dentistry, London, UK, according to manufacturer's protocol. For further details, see supplementary methods.

\section{Quantitative methylation specific PCR (qMSP)}

\section{Patient material}

Biopsy sample set: DNB specimens (formalin-fixed, paraffin-embedded (FFPE)) from patients undergoing transrectal ultrasound-guided biopsy due to suspicion of prostate cancer were obtained from Dept. of Pathology, Aarhus University Hospital, DK, as described elsewhere (Moller et al., Scientific Reports, in press). We obtained normal $(\mathrm{N})$ biopsy samples from 25 patients with exclusively cancer-negative biopsies, tumor biopsies from 25 patients diagnosed with prostate cancer (T), and histologically normal (AN) biopsies from 25 patients with prostate cancer detected in other biopsies.

Radical prostatectomy cohorts: Cohort 1 (training cohort) consisted of 566 curatively intended RP patients with histologically verified clinically localized prostate cancer (FFPE) from the Dept. of Urology, Aarhus University Hospital, DK (collected 1997-2009). All specimens were assessed by a trained pathologist. Punch biopsies were obtained from FFPE block-areas with $>$ $80 \%$ cancer cells, as described previously $[9,14,36]$. Of the 566 patients, 1 withdrew consent, 37 were either lost to follow-up, had $<3$ months follow-up, or suffered BCR within 3 months post-RP, 42 were excluded because of pre-/postoperative endocrine treatment or missing data for endocrine treatment, 6 were excluded due to positive lymph node status, 1 lacked clinical data, and 240 were excluded due to lack of tissue in the FFPE block or insufficient DNA concentration/quality. Final analyses comprised 203 prostate cancer samples (Table 1).

Cohort 2 consisted of 448 and 117 curatively intended RP patients with histologically verified, clinically localized prostate cancer (FFPE) from Moffitt Cancer Center, U.S. (collected 1987-2006) and University Hospital Zurich, Switzerland (collected 1993-2001). Of the 565 patients, 19 were either lost to follow-up, had < 3 months follow-up, or suffered BCR within 3 months post-RP, 82 were excluded because of pre-/postoperative endocrine treatment or missing data for endocrine treatment, 6 were excluded due to positive lymph node status, 13 were excluded due to ethnicity (non-European descent), 118 lacked clinical data, and 41 lacked tissue in the FFPE block or had insufficient DNA concentration/ quality. Final analyses comprised 286 samples (Table 1). See Figure S8A for flow chart of inclusion/exclusion criteria for both cohorts according to REMARK guidelines [37]. The compositions of the final cohorts were similar to that of the original cohorts (Table S12).

FFPE AN tissue samples from RP specimens and benign prostate hyperplasia (BPH) specimens (transurethral resection of the prostate) were sampled as described above for cohort 1 (Table 1, Figure S8B).

\section{DNA purification and bisulfite conversion}

For DNA purification from DNBs, $3-\mu \mathrm{m}$ tissue sections were deparaffinized and DNA was extracted using the QIAamp DNA FFPE Tissue Kit (Qiagen) according to manufacturer's protocol. DNA from RP specimens (punch biopsies) was extracted in Denmark and Switzerland using gDNA Eliminator columns from the RNeasy plus micro kit (Qiagen) and the blood and cell culture DNA kit (Qiagen), respectively, as previously described [9]. DNA from FFPE samples from the U.S. was extracted using the QIAamp DNA FFPE tissue kit (Qiagen). All DNA samples were bisulfite-converted using the EZ-96 DNA MethylationGold KitTM (Zymo research).

qMSP assays (Table S13) were designed using Primer3Plus [38] and Beacon DesignerTM (Premier Biosoft) and run as previously described [9]. Briefly, 5 ng bisulfite-converted DNA was analyzed per reaction. DNA and mastermix (Taqman universal mastermix no UNG and primer/probe sets) were run in 384-well plates on the Applied Biosystems 7900HT real-time thermal cycler. Bisulfite-converted and un-converted CpGenome Universal Methylated DNA (Millipore), 2 negative controls (whole-genome amplified DNA and $\mathrm{H}_{2} \mathrm{O}$ ), as well as serially diluted methylated DNA samples for standard curve analysis, were included on each plate. All reactions were run in triplicates. MYOD1 and aluC4 served as controls for DNA quality/quantity [9, 39], and aluC4 was used for normalization. Samples were excluded from further analysis if $\geq 2$ aluC4 reactions had $\mathrm{Ct}>25$ and/ or $M Y O D 1$ was not amplified. Samples were considered negative for methylation if $\geq 2$ methylation-specific reactions did not amplify. Bisulfite-converted DNA from DNBs was pre-amplified prior to qMSP (Table S13).

\section{Statistics}

Statistical analyses were performed using STATA v. 11.2 (StataCorp, College Station, TX, USA). Associations between DNA methylation and clinicopathological variables were assessed by Wilcoxon rank-sum test and Spearman correlations. BCR (defined as PSA $\geq 0.2$ $\mathrm{ng} / \mathrm{ml}$ ) was the clinical endpoint in survival analyses. Patients without BCR were censored at their last normal PSA measurement. In all cases, $p<0.05$ was considered significant. Where appropriate, correction for multiple testing was conducted according to Hochberg [40]. The prognostic potential of methylation marker candidates was analyzed by uni- and multivariate Cox regression analysis, Kaplan-Meier analysis and two-sided log-rank tests. Predictive accuracy was estimated using Harrell's C-index 
[41]. For multivariate testing, all clinicopathological parameters significant in univariate analysis were included. SM status was unavailable for U.S. patients, and was therefore excluded from multivariate analysis in cohort 2 .

Prognostic 2-gene model: For each gene, patients in cohort 1 (training cohort) were dichotomized into high/low-methylation groups by ROC analysis of BCR status at 36 months follow-up. Cohort 2 patients were dichotomized by the exact numerical cutoff values defined in cohort 1 (RHCG: $\geq 0.3608787$; TCAF1: $\geq 0.519267)$. For the dichotomized 2-gene panel (dRHCG-TCAF1), patients were included in the high-methylation group if both genes were highly methylated. For the trichotomized 2-gene panel (tRHCG-TCAF1), patients were included in the high-methylation group if both genes were highly methylated, the intermediate group if one gene was highly methylated, and the low-methylation group if neither gene was highly methylated.

\section{Bisulfite sequencing, RNA-seq, external datasets}

See Supplementary Materials and Methods.

\section{Abbreviations}

450K: Illumina Infinium HumanMethylation450 BeadChip; AN: Adjacent normal; AUC: Area under the curve; BCR: Biochemical recurrence; BPH: Benign prostate hyperplasia; BS: Bisulfite sequencing; CGI: $\mathrm{CpG}$ island; CI: Confidence interval; DMC: Differentially methylated CpG site; DNB: Diagnostic needle biopsy; FFPE: Formalin-fixed, paraffin-embedded; GS: Gleason score; N: Normal; NM: Non-malignant; PSA: Prostatespecific antigen; pT: Pathological tumor stage; qMSP: Methylation-specific qPCR; ROC: Receiver operating characteristics; RP: Radical prostatectomy; SM: Surgical margin; TCGA: The Cancer Genome Atlas; TSG: Tumor suppressor gene; TSS: Transcription start site

\section{ACKNOWLEDGMENTS}

We thank Margaret Gellett, Birgitte Trolle, Louise Nielsen, Maria Engtoft Skjott, and Pamela Celis for excellent technical assistance. The Danish Cancer Biobank (DCB) is acknowledged for biological material. The results presented here are in part based upon data generated by the TCGA Research Network: http:// cancergenome.nih.gov.

\section{COMPETING INTERESTS}

The authors declare no potential conflicts of interest.

\section{FUNDING}

This work was supported by Innovation Fund Denmark, the Danish Cancer Society and Aarhus University (fellowship for SHS) and, in part, by the National Cancer Institute (R01CA128813, PI: JYP). Author contributions: Conception and design: KDS, SHS. Development of methodology: SHS, CH, MM, MS, JSP. Acquisition of data: SHS, TMS, JYP, PW, SH, MM, $\mathrm{JH}, \mathrm{IN}$. Analysis and interpretation of data: SHS, MS, KDS. Writing of the manuscript: SHS, KDS. Revision and approval of the final manuscript: All authors. Study supervision: KDS, TFO.

\section{Data and materials availability}

Data is available upon request.

\section{Editorial note}

This paper has been accepted based in part on peerreview conducted by another journal and the authors' response and revisions as well as expedited peer-review in Oncotarget.

\section{REFERENCES}

1. International Agency for Research on Cancer, Who. Globocan 2012. http://globocaniarcfr/Pages/fact sheets canceraspx.

2. Baylin SB, Jones PA. A decade of exploring the cancer epigenome - biological and translational implications. Nat Rev Cancer. 2011; 11: 726-34. doi: 10.1038/nrc3130.

3. Strand SH, Orntoft TF, Sorensen KD. Prognostic DNA methylation markers for prostate cancer. Int J Mol Sci. 2014; 15: 16544-76. doi: 10.3390/ijms150916544.

4. Jeanmougin M, de Reynies A, Marisa L, Paccard C, Nuel G, Guedj M. Should we abandon the $t$-test in the analysis of gene expression microarray data: a comparison of variance modeling strategies. PLoS One. 2010; 5: e12336. doi: 10.1371/journal.pone.0012336.

5. Chiam K, Ricciardelli C, Bianco-Miotto T. Epigenetic biomarkers in prostate cancer: Current and future uses. Cancer Lett. 2014; 342: 248-56. doi: 10.1016/j. canlet.2012.02.011.

6. Zhu Y, Qiu P, Ji Y. TCGA-assembler: open-source software for retrieving and processing TCGA data. Nat Methods. 2014; 11: 599-600. doi: 10.1038/nmeth.2956.

7. Zhu Y, Xu Y, Helseth DL, Jr., Gulukota K, Yang S, Pesce LL, Mitra R, Muller P, Sengupta S, Guo W, Silverstein JC, Foster I, Parsad N, et al. Zodiac: A Comprehensive Depiction of Genetic Interactions in Cancer by Integrating TCGA Data. J Natl Cancer Inst. 2015; 107. doi: 10.1093/ jnci/djv129. 
8. Lowe R, Rakyan VK. Marmal-aid - a database for Infinium HumanMethylation450. BMC Bioinformatics. 2013; 14: 359. doi: 10.1186/1471-2105-14-359.

9. Haldrup C, Mundbjerg K, Vestergaard EM, Lamy P, Wild P, Schulz WA, Arsov C, Visakorpi T, Borre M, Hoyer S, Orntoft TF, Sorensen KD. DNA methylation signatures for prediction of biochemical recurrence after radical prostatectomy of clinically localized prostate cancer. J Clin Oncol. 2013; 31: 3250-8. doi: 10.1200/JCO.2012.47.1847.

10. Paziewska A, Dabrowska M, Goryca K, Antoniewicz A, Dobruch J, Mikula M, Jarosz D, Zapala L, Borowka A, Ostrowski J. DNA methylation status is more reliable than gene expression at detecting cancer in prostate biopsy. Br J Cancer. 2014; 111: 781-9. doi: 10.1038/bjc.2014.337.

11. Trock BJ, Brotzman MJ, Mangold LA, Bigley JW, Epstein JI, McLeod D, Klein EA, Jones JS, Wang S, McAskill T, Mehrotra J, Raghavan B, Partin AW. Evaluation of GSTP1 and APC methylation as indicators for repeat biopsy in a high-risk cohort of men with negative initial prostate biopsies. BJU Int. 2012; 110: 56-62. doi: 10.1111/j.1464410X.2011.10718.X.

12. Weiss G, Cottrell S, Distler J, Schatz P, Kristiansen G, Ittmann M, Haefliger C, Lesche R, Hartmann A, Corman $\mathrm{J}$, Wheeler T. DNA methylation of the PITX2 gene promoter region is a strong independent prognostic marker of biochemical recurrence in patients with prostate cancer after radical prostatectomy. J Urol. 2009; 181: 1678-85. doi: 10.1016/j.juro.2008.11.120.

13. Banez LL, Sun L, van Leenders GJ, Wheeler TM, Bangma $\mathrm{CH}$, Freedland SJ, Ittmann MM, Lark AL, Madden JF, Hartman A, Weiss G, Castanos-Velez E. Multicenter clinical validation of PITX2 methylation as a prostate specific antigen recurrence predictor in patients with postradical prostatectomy prostate cancer. J Urol. 2010; 184: 149-56. doi: 10.1016/j.juro.2010.03.012.

14. Kristensen H, Haldrup C, Strand S, Mundbjerg K, Mortensen MM, Thorsen K, Ostenfeld MS, Wild PJ, Arsov C, Goering W, Visakorpi T, Egevad L, Lindberg J, et al. Hypermethylation of the GABRE miR-452 miR-224 promoter in prostate cancer predicts biochemical recurrence after radical prostatectomy. Clin Cancer Res. 2014; 20: 2169-81. doi: 10.1158/1078-0432.CCR-13-2642.

15. Steyerberg EW, Vickers AJ, Cook NR, Gerds T, Gonen M, Obuchowski N, Pencina MJ, Kattan MW. Assessing the performance of prediction models: a framework for some traditional and novel measures. Epidemiology (Cambridge, Mass). 2010; 21: 128-38. doi: 10.1097/ EDE.0b013e3181c30fb2.

16. Pencina MJ, D'Agostino RB, Sr., D'Agostino RB, Jr., Vasan RS. Evaluating the added predictive ability of a new marker: from area under the ROC curve to reclassification and beyond. Stat Med. 2008; 27: 157-72; discussion 207-12. doi: 10.1002/sim.2929.

17. Cooperberg MR, Davicioni E, Crisan A, Jenkins RB, Ghadessi M, Karnes RJ. Combined value of validated clinical and genomic risk stratification tools for predicting prostate cancer mortality in a high-risk prostatectomy cohort. Eur Urol. 2015; 67: 326-33. doi: 10.1016/j. eururo.2014.05.039.

18. Klein EA, Cooperberg MR, Magi-Galluzzi C, Simko JP, Falzarano SM, Maddala T, Chan JM, Li J, Cowan JE, Tsiatis AC, Cherbavaz DB, Pelham RJ, Tenggara-Hunter I, et al. A 17-gene assay to predict prostate cancer aggressiveness in the context of Gleason grade heterogeneity, tumor multifocality, and biopsy undersampling. Eur Urol. 2014; 66: 550-60. doi: 10.1016/j.eururo.2014.05.004.

19. Freedland SJ, Gerber L, Reid J, Welbourn W, Tikishvili E, Park J, Younus A, Gutin A, Sangale Z, Lanchbury JS, Salama JK, Stone S. Prognostic utility of cell cycle progression score in men with prostate cancer after primary external beam radiation therapy. Int J Radiat Oncol Biol Phys. 2013; 86: 848-53. doi: 10.1016/j.ijrobp.2013.04.043.

20. Koksal IT, Ozcan F, Kadioglu TC, Esen T, Kilicaslan I, Tunc M. Discrepancy between Gleason scores of biopsy and radical prostatectomy specimens. Eur Urol. 2000; 37 : 670-4. doi: 20216.

21. Chen BS, Xu ZX, Xu X, Cai Y, Han YL, Wang J, Xia SH, $\mathrm{Hu} \mathrm{H}$, Wei F, Wu M, Wang MR. RhCG is downregulated in oesophageal squamous cell carcinomas, but expressed in multiple squamous epithelia. Eur J Cancer. 2002; 38: 1927 36.

22. Han KH, Croker BP, Clapp WL, Werner D, Sahni M, Kim J, Kim HY, Handlogten ME, Weiner ID. Expression of the ammonia transporter, rh C glycoprotein, in normal and neoplastic human kidney. J Am Soc Nephrol. 2006; 17: 2670-9. doi: 10.1681/asn.2006020160.

23. Biver S, Belge H, Bourgeois S, Van Vooren P, Nowik M, Scohy S, Houillier P, Szpirer J, Szpirer C, Wagner CA, Devuyst O, Marini AM. A role for Rhesus factor Rhcg in renal ammonium excretion and male fertility. Nature. 2008; 456: 339-43. doi: 10.1038/nature07518.

24. Gkika D, Lemonnier L, Shapovalov G, Gordienko D, Poux C, Bernardini M, Bokhobza A, Bidaux G, Degerny C, Verreman K, Guarmit B, Benahmed M, de Launoit Y, et al. TRP channel-associated factors are a novel protein family that regulates TRPM8 trafficking and activity. J Cell Biol. 2015; 208: 89-107. doi: 10.1083/jcb.201402076.

25. Asuthkar S, Elustondo PA, Demirkhanyan L, Sun X, Baskaran P, Velpula KK, Thyagarajan B, Pavlov EV, Zakharian E. The TRPM8 protein is a testosterone receptor: I. Biochemical evidence for direct TRPM8-testosterone interactions. J Biol Chem. 2015; 290: 2659-69. doi: 10.1074/jbc.M114.610824.

26. Liu Z, Wu H, Wei Z, Wang X, Shen P, Wang S, Wang A, Chen W, Lu Y. TRPM8: a potential target for cancer treatment. Journal of Cancer Research and Clinical Oncology. 2016: 1-11. doi: 10.1007/s00432-015-2112-1.

27. Maunakea AK, Nagarajan RP, Bilenky M, Ballinger TJ, D'Souza C, Fouse SD, Johnson BE, Hong C, Nielsen C, Zhao Y, Turecki G, Delaney A, Varhol R, et al. Conserved 
role of intragenic DNA methylation in regulating alternative promoters. Nature. 2010; 466: 253-7. doi: 10.1038/ nature09165.

28. Jones PA. Functions of DNA methylation: islands, start sites, gene bodies and beyond. Nat Rev Genet. 2012; 13: 484-92. doi: 10.1038/nrg3230.

29. Yi JM, Tsai HC, Glockner SC, Lin S, Ohm JE, Easwaran H, James CD, Costello JF, Riggins G, Eberhart CG, Laterra J, Vescovi AL, Ahuja N, et al. Abnormal DNA methylation of CD133 in colorectal and glioblastoma tumors. Cancer Res. 2008; 68: 8094-103. doi: 10.1158/0008-5472.can-07-6208.

30. Missol-Kolka E, Karbanova J, Janich P, Haase M, Fargeas CA, Huttner WB, Corbeil D. Prominin-1 (CD133) is not restricted to stem cells located in the basal compartment of murine and human prostate. Prostate. 2011; 71: 254-67. doi: 10.1002/pros.21239.

31. Dehan P, Waltregny D, Beschin A, Noel A, Castronovo V, Tryggvason K, De Leval J, Foidart JM. Loss of type IV collagen alpha 5 and alpha 6 chains in human invasive prostate carcinomas. Am J Pathol. 1997; 151: 1097-104.

32. Li P, Xu G, Li G, Wu M. Function and mechanism of tumor suppressor gene LRRC4/NGL-2. Mol Cancer. 2014; 13: 266. doi: 10.1186/1476-4598-13-266.

33. Zhang Z, Li D, Wu M, Xiang B, Wang L, Zhou M, Chen P, Li X, Shen S, Li G. Promoter hypermethylation-mediated inactivation of LRRC4 in gliomas. BMC Mol Biol. 2008; 9: 99. doi: 10.1186/1471-2199-9-99.

34. Strausberg RL, Feingold EA, Grouse LH, Derge JG, Klausner RD, Collins FS, Wagner L, Shenmen CM, Schuler GD, Altschul SF, Zeeberg B, Buetow KH, Schaefer CF, et al. Generation and initial analysis of more than 15,000 full-length human and mouse cDNA sequences. Proc Natl Acad Sci U S A. 2002; 99: 16899-903. doi: 10.1073/ pnas.242603899.
35. Albertsen PC, Hanley JA, Fine J. 20-year outcomes following conservative management of clinically localized prostate cancer. JAMA. 2005; 293: 2095-101. doi: 10.1001/ jama.293.17.2095.

36. Kristensen H, Thomsen AR, Haldrup C, Dyrskjot L, Hoyer S, Borre M, Mouritzen P, Orntoft TF, Sorensen KD. Novel diagnostic and prognostic classifiers for prostate cancer identified by genome-wide microRNA profiling. Oncotarget. 2016; 7: 30760-71. doi: 10.18632/ oncotarget.8953.

37. McShane LM, Altman DG, Sauerbrei W, Taube SE, Gion M, Clark GM. REporting recommendations for tumour MARKer prognostic studies (REMARK). Eur J Cancer. 2005; 41: 1690-6. doi: 10.1016/j.ejca.2005.03.032.

38. Untergasser A, Nijveen H, Rao X, Bisseling T, Geurts R, Leunissen JA. Primer3Plus, an enhanced web interface to Primer3. Nucleic Acids Res. 2007; 35: W71-4. doi: 10.1093/nar/gkm306.

39. Weisenberger DJ, Campan M, Long TI, Kim M, Woods C, Fiala E, Ehrlich M, Laird PW. Analysis of repetitive element DNA methylation by MethyLight. Nucleic Acids Res. 2005; 33: 6823-36. doi: 10.1093/nar/gki987.

40. Hochberg Y. A sharper Bonferroni procedure for multiple tests of significance. Biometrika. 1988; 75: 800-2. doi: 10.1093/biomet/75.4.800.

41. Harrell FE, Jr., Califf RM, Pryor DB, Lee KL, Rosati RA. Evaluating the yield of medical tests. JAMA. 1982; 247 : 2543-6. 